\title{
THE COGNITIVE NEUROSCIENCE OF MEMORY
}

What is memory? It is often associated with the "thinking of again" or "recalling to the mind" of something learned at an earlier time. Descriptions of this sort imply a conscious awareness in the rememberer that they are recollecting something of the past. For example, we might remember our first day of school or some general knowledge such as who the prime minister is. On closer reflection, this is only really the tip of the iceberg when we look at the full range of human memory capabilities. Much of our memory is submerged from conscious view (e.g., skills such as driving or typewriting). Performance on complex tasks such as playing a musical instrument can even be disrupted when conscious awareness intrudes. We learn and remember how to use language often without having to be conscious of its grammatical rules. A better description of memory could be 'the ability to retain and utilize acquired information or knowledge'.

Memory is an integral part of our existence, yet it is only vaguely understood. Through empirical studies on people, the methods of cognitive psychology have lead to some useful descriptions, distinctions and theoretical advances in our understanding of different types of memory. These approaches have paid little attention to the biological substrate of memory - the brain. This is probably partly because, until recently, the tremendous complexity of the brain has hampered our ability to gain useful insights into memory from it. But now, our understanding of the brain is beginning to reach a level of sophistication that may allow this. Tentative links between brain processes and cognitive theories are beginning to emerge in our quest to understand memory. 
This essay is about some of these tentative links between brain and mind, and how they are may set the stage for some potentially revolutionary insights into memory, not only as a brain process, but also as a psychological process. Firstly, some of the important conceptual developments in understanding memory from the methods of cognitive psychology will be summarised. The remainder of this essay will cover some of the brain mechanisms possibly involved in memory, attempting to relate them to the cognitive psychology framework. Possible mechanisms at the cellular level will be discussed, focussing on long term potentiation which has received widespread attention in physiological memory research. Cases of amnesia will be discussed and what they might imply about the specific functions in memory the relevant brain structures might perform. Models as to how these functions might be achieved will conclude this section. The following section on implicit memory completes the full circle, highlighting how research on patients with amnesia has interacted with cognitive psychological research regarding the multiple memory systems view. Finally, some of the other brain structures and mechanisms thought to be involved in different aspects of memory will be discussed. 


\section{COGNITIVE THEORIES}

One of the most important conceptual developments in cognitive theorizing is the subdivision of memory into three separate processes of encoding, storage and retrieval. Encoding, which can be further broken down into acquisition and consolidation stages, involves the laying down of a memory trace. Storage is the maintenance of a memory trace over time while retrieval is the process of reactivating a stored memory for current use. Encoding can be studied by manipulating the study conditions while participants are in the learning phase of a memory experiment. While storage is difficult to study directly it can be inferred from decay rates in retention over time. The retrieval process is the easiest to observe, often involving recall or recognition tests for previously observed stimuli. Useful theories to emerge from this approach include the encoding specificity principle and the levels of processing concept (Matlin, 1996). The encoding specificity principle states that memory retrieval is best in conditions, both internal and external, that match encoding conditions the closest. The levels of processing theory suggests that deep, meaningful and elaborative encoding leads to the best memory retrieval later on. 
Another important conceptual development is the division of memory into three different stages of sensory, short term and long term memory. Sensory memory serves to briefly hold stimuli in a raw (sensory) form so that you can register and attend to it. Ichonic (visual) sensory memory can be experimentally studied by using the partial report technique. This involves flashing an array of letters and subsequently directing participants to name the letters on a particular row. While participants cannot name the whole array they can easily name any row immediately requested - suggesting the whole image is stored briefly (1-2 seconds) (Gazzaniga, Ivry \& Mangun, 1998). Short term memory (STM) is a limited capacity store that, for example, can be assessed with a digit span task. Most people can hold between five and nine items in STM, but by 'chunking' items together can hold more information. Without rehearsal, this memory trace fades over the course of minutes. A current debate is about how much of this is due to decay alone or interference from subsequent stimuli. Recall for items often shows a J-shaped 'serial position curve' where early and late items presented in a list are recalled better. Late item success is known as the recency effect and reflects short term memory fading, while early item success is known as the primacy effect and is though to reflect a long term store (Matlin, 1996). Long term memory (LTM) is a more permanent, apparently limitless store, containing all our knowledge of the world and memories of the past. Information can be difficult to retrieve from long term storage - retrieval cues need to closely match the way the memory was encoded into LTM.

Baddeley (1995) uses the term 'working memory' rather than STM to describe the memory system that allows us to hold and manipulate stored information 'on-line'. Working memory allows relevant information from our long term store to be brought in and used with current mental processing. It is not unitary, rather it is divided into 
three subsystems - the central executive and its' two slave systems, the phonological loop and the visuo-spatial sketch-pad. The central executive is like the 'supervisory attentional system' proposed by Norman and Shallice (1986) (cited in Gazzaniga et al., 1998) - it is involved in planning and attentional control, linking the slave systems to LTM. The phonological loop serves to hold and refresh an articulation (e.g., mentally rehearsing a phone number) while the visuo-spatial sketch-pad allows location and visual information to be held. The independence of these two slave systems may help explain how we can perform well in some divided attention tasks and poorly in others - it is the limited capacity of the subsystems rather than of working memory itself that is important (Baddeley, 1995).

The division of memory into dissociable forms is another important conceptual development from cognitive psychology. Numerous facts and dissociations have been difficult to fit into a unitary view as the multiple memory systems perspective has gained acceptance. (Support from neuropsychology - especially cases of amnesia - has been important in the development of this perspective - see later.) The main distinction is between declarative and nondeclarative memory systems. This distinction is also sometimes referred to as an explicit/implicit, direct/indirect or conscious/unconscious division of memory.

Declarative memory is memory that we are conscious of and can verbalise. It can be subdivided into episodic memory (autobiographical memories, memory for particular instances of learning - including the context and source of the memory), semantic memory (facts, relations, general knowledge and understanding, not bound to a particular context) and primary or working memory (Tulving, 1995). Also known as explicit memory, it is easily tested by recall or recognition measures in an experiment. 
Nondeclarative (or implicit) memory includes classical conditioning and procedural memory (motor skills and habits such as tying up shoelaces). These forms of memory are difficult to describe verbally - they can be 'encoded' and 'retrieved' with little or no conscious awareness of it. Unlike declarative memory, nondeclarative memory is inflexible and bound to the modalility of the original response systems (Squire \& Knowlton, 1995). Schacter (1995) defines implicit memory as the facilitation of performance on a task due to previous experience without conscious recollection or awareness of that experience. Priming is an important form of implicit memory that has received much attention in recent years. It involves the facilitated perception of stimuli due to pre-exposure to them, without conscious awareness that the stimuli had been perceived earlier. The fact that amnesic patients show preserved priming is of strong interest in cognitive neuroscience (a more detailed discussion will come later).

Cognitive psychology has brought about some useful conceptual developments toward a more complete understanding of memory. Bringing these ideas together, further progress could be made by considering how the processes of encoding, storage and retrieval operate in the different memory systems of sensory, working memory and the declarative and nondeclarative forms of LTM. How independent are the processes of encoding, storage and retrieval? How do the dissociable memory systems interact? Are these divisions accurate? - perhaps we should be looking for more functional divisions rather than task divisions. If these divisions are accurate there should be neural correlates of them in the brain. Are we ready to start understanding correlations between neural mechanisms and memory processes? How well does neuroscientific data support cognitive theories of memory? At the lower level of analysis of individual neurons, some interesting insights into how the brain enables memory have been found. 


\section{THE CELLULAR BASIS OF MEMORY}

How might memory be represented at the neuronal level? Presumably, connections between neurons are somehow altered in response to some experience and the persistence of this change enables memory. The time-frame of these changes may provide some clues about differences between short and long-term memory (if this is a valid distinction). A stimulus to be remembered might activate a collection of neurons. Synaptic changes must somehow bind this collection together so that at least part of the same collection can be simultaneously reactivated at a later time.

Hebb (1949) (cited in Kalat, 1998) proposed a mechanism by which learning can occur at the neuronal level: If there is presynaptic and postsynaptic activity occurring at the same time, for whatever reason, the connection between these cells strengthens. This mechanism could allow for associative learning to occur between previously unrelated stimuli. For example, in classical conditioning, if neural activity representing a conditioned stimulus (e.g., bell) occurs simultaneously with activity representing an unconditioned response (e.g., salivating, caused by the presence of food) then connections between them could strengthen via Hebbian learning. Are there cellular mechanisms that allow learning of the type proposed by Hebb? A phenomenon known as long term potentiation has created excitement as it is a suitable candidate for such a learning mechanism.

Because of its known importance to memory and ease of study, the hippocampus (a medial temporal lobe structure) has received much attention. By stimulating hippocampal slices with an electrode, downstream responses have been observed (Graham, 1990). By stimulating the perforant pathway, an excitatory postsynaptic 
potential (EPSP) - a depolarisation - can be observed in the dentate gyrus. An initial stimulus may result in a certain EPSP, but after a rapid volley of stimuli, a single stimulus has been observed to result in a significantly larger EPSP (Graham, 1990). This enhancement, which has been observed for as long as weeks after the initial volley, is known as long term potentiation (LTP). Activation of perforant presynaptic neurons occurred together with activation of postsynaptic dentate gyrus neurons (EPSPs exceeding threshold) during the volley and the EPSP of a single subsequent stimulus was enhanced. This implies that a strengthening of connections has occurred and that Hebbian learning can occur in the hippocampus. LTP has been demonstrated often and not just in the hippocampus. The next sensible question lies in what cellular mechanisms allow this to occur and on what time frame they operate.

One such mechanism involves glutamate receptors: If a depolarised postsynaptic neuron receives further glutamate, NMDA receptors can operate, opening another ion channel that allows calcium into the membrane (Winson, 1990). This influx of calcium ions can lead to LTP through a variety of mechanisms. One of the quickest mechanisms involves the activation of calpain (a proteinase) that degrades fodrin, leading to uncovering of hidden receptors (Lynch \& Baudry, 1984). While this can occur in seconds, the breakdown of fodrin may also lead to changes in the dendrites' cytoskeleton after about ten minutes that may change dendritic spines into shorter, more stubby shapes (Graham, 1990). Shorter spines are more conducive to the transfer of depolarisation towards the cell body - increasing response to the presynaptic message. This change may last for several hours before fading. Influx of calcium decreases its concentration outside that may lead to polymerisation of ependymins (a protein). Graham (1990) argues that this could create a matrix that guides synaptic growth, strengthening weak associated synapses. This protein matrix 
may also act to guide sprouting. Changes of this sort may take several hours to initiate but last for days or longer. (These time estimates are based on the time frame of learning impairments, often observed in animals, of experimental interventions that impede these biochemical pathways - e.g., protein synthesis inhibitors.)

Another mechanism for synaptic plasticity including LTP involves a second messenger called cyclic AMP (Graham, 1990). Receptor activation leads to cyclic AMP activation - it can generate EPSPs by opening ion channels. Because it can persist for several seconds in the postsynaptic membrane this mechanism could aid short term memory. Cyclic AMP can also trigger a cascade of chemical reactions via protein kinase and CREB (cyclic AMP response element binding protein) that allows gene expression through protein synthesis (Yin \& Tully, 1998). Such protein synthesis could lead to axon sprouting and extra dendritic branching that enables new connections to be formed between cells as well as strengthening old ones. Such changes could support a more permanent long term type of memory. It has been suggested that the brain mechanisms (including survival (trophic) factors) important in the development of the infant brain can also allow for synaptic plasticity in the adult brain (Black, 1995).

How do these cellular mechanisms relate to the conceptual developments in the cognitive psychology of memory? Bailey and Kandel (1995) make a distinction between shorter term forms of memory mediated by changes in the effectiveness of preexisting synaptic connections and longer term forms due to establishment of new connections mediated by protein synthesis. However, much of this knowledge has been gained from animal studies without the functional memory organization that humans have. It is quite possible that these mechanisms (and many more) operate in humans, and that protein synthesis mediated changes are viable candidates for LTM 
due to their more permanent effects. However, there does not seem to be a sharp definition in the short term realm - many different mechanisms of different time scales appear to be operating. Graham (1990) proposes that there should at least be an intermediate term memory between STM and LTM, if not a finer grading. Encoding specificity could be interpreted at the cellular level - the closer the external and internal cues are to the conditions at encoding, the closer the currently activated collection of neurons are to that of the memory trace. This would increase the likelihood of reactivating the enhanced connections of the memory trace.

How do these cellular processes occur in the context of natural brain functioning? Attention should be drawn towards linking this cellular knowledge to the higher level of analysis of functional organization of the brain. Different memory systems may involve different mechanisms of synaptic plasticity. Sensory memory might not involve any synaptic plasticity - perhaps it reflects the decay of a reverberating circuit that is set up in perception itself. Working memory could also involve a prefrontally refreshed frontal-posterior loop (see later) - such rehearsal may help ensure the sufficient synaptic facilitation occurs so that independent, longer term connections can develop. Cellular level analyses of memory may provide some insights into the storage process, but on their own cannot explain encoding or retrieval. Cellular level analysis alone provides little indication of the dissociations observed between different memory systems in cognitive psychology. Investigating the possible functional specializations of brain regions and circuits has proved fruitful in this regard. 


\section{THE MEDIAL TEMPORAL LOBE AND AMNESIA}

The case of H.M., who had his hippocampus bilaterally removed to treat epilepsy, has been well studied (Gazzaniga et al., 1998). As a result, he suffered from amnesia which has prevented formation of new long term memories. For example, after teeing off in golf he would forget that he had done so and tee off again or he would quickly forget where he hit the ball (Schacter, 1996). He would meet a person he had talked to, at length, the day before and have no recollection of ever seeing him before. The inability to form new long term memories is known as anterograde amnesia and is a classic symptom of patients with damage to their medial temporal lobes. Converging evidence from PET studies also point to the importance of this region in memory (Gazzaniga et al., 1998). Retrograde amnesia is lost memory for times before the incident (e.g., head trauma) that lead to the amnesia. In amnesics like H.M., retrograde amnesia is often limited or temporally graded to less than a few years before the brain damage (Squire \& Alvarez, 1998). Patients have good memory for the distant past but for events closer to their 'incident' their memory declines. Despite such obvious deficits, patients show normal IQ and short term memory (e.g., on a digit span task). Patients with Korsakoff's syndrome (damage to the dorsomedial thalamus due to vitamin deficiency) also suffer a similar pattern of memory deficits. This may make sense since there are close medial temporal lobe to diencephalic connections and they can be treated as a single functional structure (Squire \& Knowlton, 1995).

What do these data from these amnesic cases suggest about the functional role of these brain structures? Consolidation of newly forming long term memories has become a strong theory (Gazzaniga et al., 1998). This interpretation is consistent with 
the anterograde and temporally graded retrograde amnesia pattern seen so often. It has been suggested that the medial temporal lobe has only a temporary role in forming LTM (Squire \& Knowlton, 1995). Squire and Alvarez (1998) suggest that consolidation is the process by which memory becomes independent of hippocampal regions. If this is so, where might consolidated LTM be stored? The neocortex has been suggested with some evidence for the lateral temporopolar and lateral inferior prefrontal cortex regions (Markowitsch, 1995). Cases (rare) of ungraded retrograde amnesia and preserved anterograde memory have involved damaged anterior lateral temporal lobes suggesting them as possible long term memory storage sites (Squire \& Alvarez, 1998). Is the medial temporal lobe (MTL) system the exclusive site for LTM until cortical representations develop? A better interpretation may be that the MTL does not store the memories themselves, but simply mediates between cortical sites. The MTL might store conjunctions tying distributed memory storage sites until adequate cortico-cortical connections become established. By using this interpretation, developed from neuropsychology case studies, links to cellular level theories of memory can be attempted through neural network models.

Squire and Alvarez (1998) propose that distributed cortical memory storage sites are slow to establish connections. Hebbian learning could cause these sites to wire together through LTP, but the sites need to be firing together on many occasions over a long time for this to establish. Hippocampo-cortical connections could establish much more quickly and intra hippocampal connections could connect respective hippocampocortical loops, thus linking distributed cortical memory sites. Reactivation of the cortical sites via hippocampal activity could create enough cortical 'firing together' occasions to enable cortico-cortical connections to establish. Winson (1990) proposes that during REM sleep (dreaming), this type of LTM consolidation occurs. 
Neural network models following this basic framework have been developed (Levine, Long \& Parks, 1998). According to one model, ‘self organization' allows the hippocampus to form a compressed representation of cortical activity and 'autoassociation' allows intrahippocampal connections to develop. A more detailed model looks within hippocampal structures and incorporates cholinergic modulation via the medial septum in distinguishing between encoding and recall. If the general framework of these models is correct, then why is the anterior lateral temporal lobe associated with older established memories? Perhaps this region is a more durable cortical mediator between distributed memory systems just as the MTL might be a more transient subcortical mediator.

Cognitive psychology distinguishes between different memory systems - how does the function of the MTL relate to these? MTL amnesias have provided a thrust towards the development of the memory systems view in cognitive psychology. The types of memory referred to above have been the explicit forms. Amnesics show preserved implicit forms of memory despite a complete lack of conscious awareness of ever encountering anything before.

\section{IMPLICIT MEMORY AND AMNESIA}

Amnesic patients can still learn in some ways. Procedural learning (such as learning a motor sequence) is intact and they can learn artificial grammar (Gazzaniga et al., 1998). In fact, people with intact brains can learn artificial grammar without explicit awareness of the rules. Fairly recently, a form of implicit memory known as priming has received widespread study in cognitive psychology (Schacter, 1987). Typically, participants view some words in an incidental learning task and are later asked to 
complete an independent word fragment test. Implicit memory is reflected by facilitated completion of words that participants were pre-exposed to, without an awareness that the pre-exposed words were relevant to the current test. One of the problems with such research is knowing whether some explicit memory is contaminating the results (Rugg, 1995). Amnesic patients are ideal for this sort of testing because they have no explicit memory for being pre-exposed at all. They show the same level of priming memory as do normal healthy participants, yet if given a recognition or recall test for the pre-exposed words, perform far worse than their counterparts. Their priming performance is dissociated from recall and recognition (Schacter, 1995).

Similar dissociations have been observed within normal participants as a function of the instructions given during pre-exposure. While recognition has been found to be improved by generating rather than simply viewing words (a deeper level of processing), perceptual priming has shown the opposite effect (Roediger, 1990). It seems that seeing the word is important for priming to occur. This has lead to the concept of a perceptual representation system (PRS) for this form of priming (Ochsner, Chiu, \& Schacter, 1998). Priming shows some perceptual specificity effects - simply changing the case that words are presented in leads to a reduction in priming. This kind of specificity has also been observed in the auditory domain (Church \& Schacter, 1994) with changes in fundamental frequency or voice intonation reducing facilitated perception of degraded spoken words. Priming of these sorts is said to be data driven and seems to be strongly intertwined with the perceptual process itself. Perhaps receptors are uncovered or some other shorter term form of LTP occurs a few levels up in the perceptual hierarchy. The nodes for these words may be primed up to be more readily activated on re-presentation of the word. There are also concept 
driven forms of priming - for example, presentation of words with negative connotations has been shown to negatively bias participants ratings of neutrally presented people in a later task (Roediger, 1990). Research on priming has regenerated interest in the possible role of unconscious processes (Jacoby, Lindsay \& Toth, 1992; Loftus \& Klinger, 1992).

Do the dissociations between implicit and explicit memory observed in amnesics and normal participants provide solid justification for such a division, with the explicit memory system dependent on the medial temporal lobes? A case of a patient with preserved recall but poor visual priming provides a double dissociation (Gazzaniga et al., 1998). The damaged area was in the extrastriate cortex (which makes sense in terms of the PRS concept). Arguing against this rigid division, the above completion of the double dissociation is tentative at best. Also, amnesics do not show quite the same perceptual specificity effects in priming tasks as normal participants do (Schacter, 1995). Explicit memory probably relies on some implicit forms of memory, but implicit memory, by definition, does not depend on explicit memory. What is it that makes us conscious that we are remembering something? Is it an overwhelming sense of familiarity or does some sort of affect tag connected to it (from the limbic system) indicate this to us.

The flow of information in memory processes is not restricted to the medial temporal lobes: Because we attribute something as a memory indicates some frontal lobe involvement. Indeed, there are direct connections between the diencephalic memory structures and frontal lobes. What about other forms of memory such as working memory and skill learning and the sometimes powerful role of emotions? How might they be manifest in the brain? 


\section{OTHER BRAIN STRUCTURES AND MEMORY}

\section{SYSTEMS}

The frontal lobes have intricate connections to posterior regions including sensory and association cortices and the limbic system (Shimamura, 1995). In general, the function of this region has been summarized as effecting inhibitory control of extraneous activity and gating. The frontal lobes have been suggested as important in working memory. Broca's area has been related to the phonological loop (Gazzaniga et al., 1998): By projecting to posterior regions, the dorsolateral prefrontal cortex might keep sensory regions active so that they can be held in memory. LTM could possibly be reactivated (into working memory) by frontal projections to the lateral anterior temporal lobes. Patients with frontal lobe damage show attention and concentration deficits that can lead to poor working memory (e.g., digit span) yet they show good explicit recognition memory (Shimamura, 1995). (This is the opposite pattern to MTL patients, giving a good dissociation between working memory and LTM.) Frontal patients show poor recall - this has been explained as being due to poor search and retrieval strategies, with the inability to inhibit irrelevant memory associations (Shimamura, 1995). (Maybe this could be related to Baddeley's working memory model: Recall depends on activating LTM into working memory while in recognition the stimuli enter more directly through attention). Deficits observed in elderly people also show a similar pattern including poor source memory and prospective memory. Both of these forms of memory require good search and retrieval strategies. This pattern has been interpreted in terms of prefrontal cortex decline - it is amongst the earliest brain regions to decline with natural aging (West, 1996). 
Other brain structures appear to enable some forms of memory too - dissociations between non-declarative forms of memory have been observed. Patients with Alzheimer's disease (diffuse cortical association area damage) show reduced perceptual priming and spared procedural, motor learning tasks while patients with Huntington's disease (damaged basal ganglia) show the opposite pattern (Schacter, 1995). Squire and Knowlton (1995) linked brain regions and systems to memory kinds that fit the distinctions made in cognitive psychology quite well, but note that we need to make specific identification where synaptic changes actually occur to gain further insights.

Emotions are known to sometimes have powerful effects on memory (Matlin, 1996). A traumatic event may elicit strong emotions that enable vivid memories of the event to persist well after the event. Research on flashbulb memories suggest that while the memory may be vivid it might not necessarily be very accurate (Matlin, 1996). There are important brain mechanisms that modulate memory storage in response to emotion - this can occur through stress hormones (Cahill \& McGaugh, 1998). Stress hormones increase blood sugar levels and arousal that may aid forming a memory trace (e.g., extra energy available in the brain). The hormones themselves have been shown to have modulatory roles in memory (effects of direct injections in animal learning tasks have shown that small amounts are beneficial while larger, more chronic doses are detrimental). The amygdala is a key brain region in emotional memory encoding (e.g., aversive conditioning has been shown to depend on this region (Davis, Campeau, Kim \& Falls, 1995). Activity in the amygdala has also been shown to modulate other memory systems such as the hippocampus (Cahill \& McGaugh, 1998). The above mechanisms may help explain why emotionally 
powerful events are sometimes remembered vividly, but our understanding is far form complete.

There are a multitude of brain structures and mechanisms that appear to be involved in different forms of and aspects of memory and probably many more that remain to be discovered. The linking of brain structures to memory functions is only a start - the real question lies in how these structures actually work in enabling these functions.

\section{DISCUSSION}

In this essay I have attempted to relate brain mechanisms to psychological processes involved in memory. In order to successfully find such links it becomes obvious that we need to be able to clearly conceptualise and characterise aspects of memory on a psychological basis if we are to identify them in the brain - indeed, this could conceivably be the ultimate goal. Neuroscience based research can help clarify and direct these concepts at the psychological level - for example, the simplistic division of STM and LTM needs to be modified based on cellular data. The argument for separate explicit and implicit memory systems seems to be supported by studies of amnesia. Dissociable memory systems within the implicit domain receive some support too.

But what about the explicit domain - what, exactly, are the differences between semantic and episodic memory in terms of brain functions? If explicit memory depends on the medial temporal lobe system as and integral component then do semantic memory deficits show the same pattern as episodic deficits do in amnesic patients? It would be interesting to see if semantic memory follows the same pattern of temporally graded retrograde loss as episodic memory does. (This would not be 
easy to test as it is difficult to know when patients may have learnt semantic knowledge before their brain damage.) Semantic memory is independent of the context it was learnt in, yet there would be an episodic trace of these occasions (somewhere) - what allows this divorce? Is the former cortico-cortical and the latter hippocampocortical? Perhaps there is parallel encoding into both memory systems when learning semantic associations with semantic storage outlasting episodic storage due to deep meaningful encoding. Is meaningful encoding successful because of widespread cortico-cortical connections reflecting related associations ('integration into semantic networks' (Matlin, 1996))? Should semantic memory be considered as 'affectless' and purely verbal only? The fact that the hippocampus is part of the limbic system and that information from the medial temporal lobe system reaches the orbitofrontal cortex (associated with 'affect tags' to stimuli (Gazzaniga et al., 1998)) might suggest that affect should not be ignored in explicit memory. Perhaps explicit memory could be divided into affective and verbal components that reverberate in a feedback loop, with episodic and semantic memory reflecting different manifestations of it. While these ideas are speculative, more clearly defined divisions of memory without the explicit domain may be fruitful.

In conclusion, the cognitive neuroscience approach is inspiring a deeper appreciation of what memory really is and how it works. 


\section{REFERENCES}

Baddeley, A. (1995). Working memory. In M.S. Gazzaniga (Ed.), The cognitive neurosciences (pp. 755-764). Cambridge, MA: MIT Press.

Bailey, C.H. \& Kandel, E.R. (1995). Molecular and structural mechanisms underlying long-term memory. In M.S. Gazzaniga (Ed.), The cognitive neurosciences (pp. 19-36). Cambridge, MA: MIT Press.

Black, I.B. (1995). Trophic interactions and brain plasticity. In M.S. Gazzaniga (Ed.), The cognitive neurosciences (pp. 9-17). Cambridge, MA: MIT Press.

Cahill, L. \& McGaugh, J.L. (1998). Modulation of memory storage. In L.R. Squire \& S.M. Kosslyn (Eds.), Findings and current opinion in cognitive neuroscience (pp. 85-90). Cambridge, Massachusetts: MIT Press.

Church, B.A. \& Schacter, D.L. (1994). Perceptual specificity of auditory priming: Implicit memory for voice intonation and fundamental frequency. Journal of experimental psychology: Learning, memory, and cognition, 20, 521-533. 
Davis, M., Campeau, S., Kim, M., \& Falls, W.A. (1995). Neural systems of emotion: The amygdala's role in fear and anxiety. In J.L. McGaugh, N.M. Weinberger, \& G. Lynch (Eds.), Brain and memory: Modulation and mediation of neural plasticity (pp. 3-40). Oxford: Oxford University Press

Gazzaniga, M.S., Ivry, R.B., \& Mangun, G.R. (1998). Cognitive neuroscience: The biology of the mind. London: Norton.

Graham, R.B. (1990). Physiological psychology. Belmont, Cal: Wadsworth.

Jacoby, L.L., Lindsay, D.S., \& Toth, J.P. (1992). Unconscious influences revealed: Attention, awareness, and control. American psychologist, 47, 802-809.

Kalat, J.W. (1998). Biological psychology (6 ${ }^{\text {th }}$ ed.). Belmont, Cal: Wadsworth.

Levine, D.S., Long, D.L., \& Parks, R.W. (Eds.) (1998). Fundamentals of neural network modelling: Neuropsychology and cognitive neuroscience. Cambridge, Massachusetts: MIT Press.

Loftus, E.F. \& Klinger, M.R. (1992). Is the unconscious smart or dumb? American psychologist, 47, 761-765.

Lynch, G. \& Baudry, M. (1984). The biochemistry of memory: A new and specific hypothesis. Science, 224, 1057-1064. 
Matlin, M.W. (1994). Cognition ( $3^{\text {rd }}$ ed.). Florida: Harcourt Brace.

Ochsner, K.N., Chiu, C-Y.P, \& Schacter, D.L. (1998). Varieties of priming. In L.R. Squire \& S.M. Kosslyn (Eds.), Findings and current opinion in cognitive neuroscience (pp. 99-104). Cambridge, Massachusetts: MIT Press.

Roediger, H.L. (1990). Implicit memory: Retention without remembering. American psychologist, 45, 1043-1056.

Rugg, M.D. (1995). Event relate potential studies of human memory. In M.S. Gazzaniga (Ed.), The cognitive neurosciences (pp. 789-801). Cambridge, MA: MIT Press.

Schacter, D.L. (1987). Implicit memory: History and current status. Journal of experimental psychology: Learning, memory, and cognition, 13, 501-518.

Schacter, D.L. (1995). Implicit memory: A new frontier for cognitive neuroscience. In M.S. Gazzaniga (Ed.), The cognitive neurosciences (pp. 815-824). Cambridge, MA: MIT Press.

Schacter, D.L. (1996). Searching for memory: The brain, the mind, and the past. New York: Basic Books. 
Shimamura, A.P. (1995). Memory and frontal lobe function. In M.S. Gazzaniga (Ed.), The cognitive neurosciences (pp. 803-813). Cambridge, MA: MIT Press.

Squire, L.R. \& Alvarez, P. (1998). Retrograde amnesia and memory consolidation: a neurobiological perspective. In L.R. Squire \& S.M. Kosslyn (Eds.), Findings and current opinion in cognitive neuroscience (pp. 75-83). Cambridge, Massachusetts: MIT Press.

Squire, L.R. \& Knowlton, B.J. (1995). Memory, hippocampus, and brain systems. In M.S. Gazzaniga (Ed.), The cognitive neurosciences (pp. 825-837). Cambridge, MA: MIT Press.

West, R.L. (1996). An application of prefrontal cortex function theory to cognitive aging. Psychological bulletin, 120, 272-292.

Winson, J. (November, 1990). The meaning of dreams. Scientific American, 42-48.

Yin, J.C. \& Tully, T. (1998). CREB and the formation of long term memory. In L.R. Squire \& S.M. Kosslyn (Eds.), Findings and current opinion in cognitive neuroscience (pp. 133-137). Cambridge, Massachusetts: MIT Press. 